\title{
XVI. On some electromagnetic experiments of Faraday and Plücker
}

\section{S. Tolver Preston}

To cite this article: S. Tolver Preston (1885) XVI. On some electromagnetic experiments of Faraday and Plücker, Philosophical Magazine Series 5, 19:117, 131-140, DOI:

10.1080/14786448508627655

To link to this article: http://dx.doi.org/10.1080/14786448508627655

册 Published online: 29 Apr 2009.

Submit your article to this journal $[\pi$

Џlll Article views: 3

Q View related articles $₫$ 
Electromagnetic Experiments of Faraday and Plïcker. 131

power of the gas increases continuously with the exhaustion which it undergoes, even when this is pushed as far as possible, it would be more philosophical to assume that an absolute vacuum is a conductor.

XVI. On some Electromagnetic Experiments of Faraday and Plücker. By S. Tolver Preston*.

[Plate II.] $A$ LTHOUGH it might seem late in the present day to call on the subject of magnetism deduced from experiment, yet wo know that cases admitting of a double interpretation do sometimes occur in experimental research ; and precisely on account of their extreme rarity are such cases liable to elude attention and to lead to misapprehensions. About the year 1869, when reading Faraday's 'Experimental Researches,' such an instance attracted my notice, and it recurred some years afterwards while perusing a paper of Plücker's (Pogg. Ann. Bd. lxxxvii. 1852, S. 353: "Ueber die Reciprocität der elektromagnetischen und magnetoelektrischen Erscheinungen "). The same ambiguous case occurred both to Faraday and Plücker; and neither appears to have noticed the fact of its being ambiguous (or admitting of a double interpretation). Yet the interpretation taken by them, when carried out to its logical conclusions, appears to contradict the theory of Ampere as to the similarity of the helix (solenoid) and magnet in their chief propertiesa theory which seems to have been hitherto generally accepted.

I will at once proceed to the point in question. Faraday, and also Plücker, adopted quite a peculiar view as to the behaviour of the system of force ("lines of force") about a magnet when rotated on its axis; and they set up in this way (led by the experiments) a fundamental distinction of principle between the translatory and rotatory motion of a magnet in regard to the behaviour of the field of force about it. Faraday himself calls his own view "singular." The following are the words of Faraday relating to this point:-

"When lines of force are spoken of as crossing a conducting circuit, it must be considered as effected by the translation of a magnet. No mere rotation of a bar-magnet on its axis produces any inductive effects on circuits exterior to it. The system of power about the magnet must not be considered as revolving with the magnet any more than the rays of light

* Communicated by the Author. 
which emanate from the sun are supposed to revolve with the sun. The magnet may even, in certain cases, be considered as revolving amongst its own forces, and producing a full electric effect sensible at the galvanometer." (Phil. Trans. 1852, page 31.)

Without at once going into the related practical question as to whether a magnet (the rotating globe of the earth, for instance) can, by " revolving amongst its own forces," become charged at the equator and poles with electricity of opposite sign (as Faraday* inferred from this experiment), I would only wish to call the unbiassed attention to the above distinction in principle between a translatory and a rotatory motion, as it seems to me that such a distinction (as a matter of theoretic reasoning) cannot possibly hold. For rotation is surely after all only a particular case of translation, viz. translation in a circle. When a magnet rotates on its axis, every part or magnetic point in it is translated (in a circle) about that axis. And certainly it will be conceded in regard to Faraday's analogy of the (axially) revolving or rotating sun, the same principle (whatever it was) that applied to the behaviour of the "rays of light" of the rotating sun, also must apply to the rays of light of the translated sun (in its proper motion). For we do not ask whether a given luminous point or portion of the sun's surface is moving in a curved or in a straight line in order to set fast the principle of the behaviour of the rays of light. The same reasoning must therefore apply to the magnet if Faraday's analogy is to hold at all, $i . e$. if (in regard to the inductive effect) the lines of force must be regarded as partaking of the motion of a magnet when it is translated, they must partake of its motion when it is rotated $\dagger$. This, therefore, constitutes the first theoretic objection to the view adopted by Faraday, and it seems to me to be in itself conclusive. But, for the sake of further illustration, I will point out how this view clashes with the generally accepted similarity in (at least) fundamental properties between the solenoid, or helix, and the magnet.

It seems always to have been taken for granted that when a solenoid, or a simple circular current, rotates on its axis in

* Plücker also inferred it from an analogous experiment, and remarks, "Hier tritt alsdann in den beiden Polen die positive, unter dem Aequator die negative Elektricität auf, während eine Indifferenzzone zwischen dem Aequator und jedem der beiden Pole liegt." (Pogg. Ann. 1852, p. 357.)

$\dagger$ Or, in other words, if the lines of force emitted by a given portion of a magnetic surface must be considered as partaking of the motion of that portion of surface, when it is translated in a straight line, they must equally do so when that portion of surface is translated in a curved line (by the rotation of the magnet on its axis). 
its own plane, the inductive effect depends only on the relative motion between the circular current and neighbouring conducting bodies, and does not depend on whether the circular current or the conducting bodies move. When, for example, a piece of wire $a b$ (Pl.II.fig. 1 ) is placed near a revolving solenoid or simple circular current, $s s$, it is quite indifferent, as regards the inductive effect, whether the circular current revolves about its centre (or axis), or whether, conversely, the wire ab is made to revolve about the fixed circular current, in the direction of the dotted line, so long as the relative motion between the two is the same. I only suppose that in reference to inductive effects with currents we have to do with relative, and not at all with absolute motions. If that is true, and also if the magnet is to resemble the solenoid in its clicef properties (according to the well-known theory of Amperre), then I contend that a magnet in the above case must behave in principle as a solenoid or circular current. Faraday, however (and with him Plücker), holds the opposite. Faraday expresses the view that it is not indifferent whether the magnet or the external conductor moves, that the effect is not dependent on relative motion ; in short, that " no mere rotation of a barmagnet on its axis produces any inductive effect on circuits exterior to it. The system of power about the magnet must not be considered as revolving with the magnet" (loc. cit. suprà).

I will now consider the experiment whence Faraday derived the above singular (or exceptional) view in regard to a rotating magnet. A copper disk $k$ (fig. 2) was cemented upon the end of a round bar-magnet $m$, with insulating paper intervening -or, in the words of Faraday :-

"The magnet and disk were rotated together, and collectors (attached to the galvanometer) brought in contact with the circumference and the central part of the copper plate. [In the diagram, the dotted line shows this outer circuit, with the galvanometer $g$.$] The galvanometer-needle moved as in former$ cases, and the direction of motion was the same as that which would have resulted if the copper only had revolved and the magnet been fixed. Neither was there any apparent difference in the quantity of deflection. Hence rotating the magnet causes no difference in the results, for a rotating and a stationary magnet produce the same effect upon the moving copper." (Phil. Trans. 1832, page 183.)

Here Faraday observed (beforehand) a certain deflection of the galvanometer when the copper disk was made alone to revolve through the lines of force of the fixed magnet. $\mathrm{He}$ observed the same deflection when the magnet and copper disk 
Phil . Maś. S. 5 .Vol.18. P1. II.

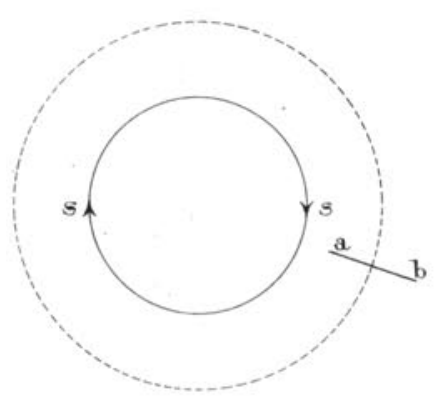

Fig. 1.

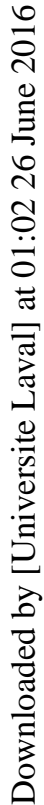

Fi .3 .

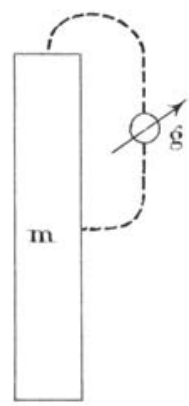



Fig. 2 .

$$
\text { Fig. 4. }
$$

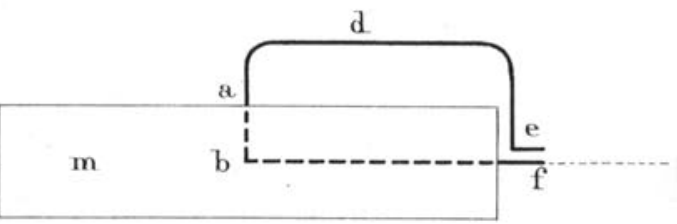

Fig. 4. (bis).

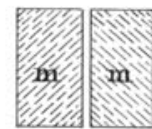

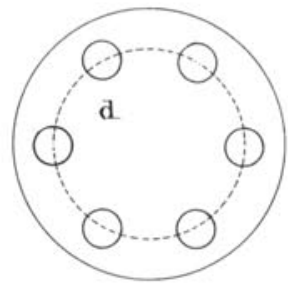

Fig. 5.

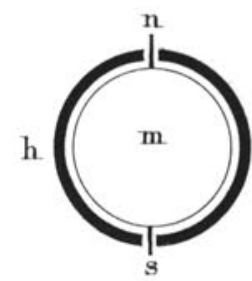

Fig. 6.

Mintern Bros, lith. 
were revolved together (as one whole). Hence he seems to bave reasoned that because the deflection remained the same, the conditions must have remained the same; and therefore that the revolving copper disk, even when attached to the magnet (and revolving with it), still continued to cut through the magnetic lines of force of the magnet, precisely as it would have done if the disk alone had revolved and the magnet been fixed. Hence Faraday concluded that the lines of force could not possibly partake of the rotatory motion of the magnet*; but that the magnet revolved "amongst its own forces."

But I believe it will be apparent here, on inspection of the diagram of the apparatus, that the same deflection would be equally consistent with the opposite supposition (viz. that the lines of force partake of the rotatory motion of the magnet, in the same sense as they partake of the translatory motion of the magnet). For, admitting that the lines of force revolve with the magnet, then they will intersect the galvanometer-loop circuit when the magnet revolves on its axis; and this will evidently produce a current of the same direction and magnitude as under Faraday's singular assumption, which he thought himself foreed into by the experiment. This, I think, is clearly one of those rare cases of a deceptive double meaning to an experimental fact, which on account of their rare occurrence are unexpected, and may consequently sometimes escape the notice of even skilful experimenters. I venture to believe that few will doubt what the true explanation here is after the above elucidation; and only in this way does the contradiction to Ampère's theory (of the similarity of the properties of the helix and magnet) vanish, as also the great theoretic difficulty of drawing a distinction between translatory and rotatory motion in regard to the behaviour of the magnetic lines of force.

I conclude, therefore, that the inferred inductive charge produced at the poles and equator of the revolving globe of the earth (as a rotating magnet), by " revolving amongst its own forces," certainly does not necessarily follow $\dagger$ from this

* After a second perfectly similar experiment to this, with a cylindrical copper cap instead of a disk, Faraday adds "Thus a singular independence of the magnetism and the bar in which it resides is rendered evident." (Page 184.)

$\dagger$ On the other hand, another quite different cause may conceivably conduce to an electric disturbance on the earth's surface, or, rather, on parts of it which are not rigidly fixed. The tides, namely, form a circular band or ring of water through which the earth revolves. These great water elevations are not therefore fixed (relatively to the revolving earth) as the glube of the earth itself is ; and consequently the magnetic lines of force carried along with the revolving globe can intersect the tides, and 
experiment. Whether there are other independent grounds for such an assumption, I am not aware, and Faraday does not state them.

It will be seen clearly that Faraday's view involves the strange assumption that although (as an admitted fact), when a copper disk is revolved parallel to and above the pole of a magnet, an inductive effect is produced on the disk [so that its centre and periphery become statically charged with electricity of opposite sign], yet when, conversely, the magnet is made to revolve with the same relative velocity below the fixed disk, no effect is produced; yet the relative motion is really the same in both cases. In fact, Faraday's aspect of the case involves the supposition that the parts of a body at rest (relatively to the body itself, when it revolves) can produce an inductive effect on the body.

The following experiment (Phil. Trans. 1832) may possess an interest, as it was considered by Faraday to confirm his view of the static charge produced at the equator and poles of a magnet by its own revolution, and which also led (Plücker especially) to speculations over the cause of the aurora borealis (Pogg. Ann. 1852, p. 357). A cylindrical bar-magnet, $m$ (fig. 3), was set by Faraday in axial rotation, and the galvanometer wires were maintained in sliding contact with the equator and one pole of the magnet respectively, as the dotted lines in the diagram indicate. A current was observed; and the cause of this current was referred (in the same way) to a static charge produced by the magnet eutting through its own (internal) lines of force, these lines, or the internal field of force, having been supposed to remain rigidly at rest, while the magnet revolved independently through it. But it will be seen that the current may be simply referred (in an analogous way to the previous example of the copper disk) to the lines of force moving with the revolving magnet, and intersecting the external circuit formed by the galvanometer wires. This explanation has the additional advantage of bringing the current formed, whether by the rotation of the magnet on its axis, or by the (inverse) revolution of the wire about the magnet, under the same cause (and not under different causes).

so cause a disturbance of the electric equilibrium on the earth's surface of the character imagined by Faraday, and assigned by him to a quite different cause. Also the trade winds, which, somewhat similarly, form a kind of air-belt through which the earth revolves, cin conceivably conduce in the same way to an electric disturbance, especially as these winds near the equator are laden with moisture, so as to become in some sense conductors. 
Twenty years afterwards, in 1852 , in order to test the case further, the following experiment was made by Faraday. An insulated wire was bent into the form of a rectangle, $e d a b f$ (fig. 4), and pushed into the slit or interval between two barmagnets of oblong cross section, of which fig. 4 (bis) shows the end view. The part of the wire buried between the magnets is dotted in the upper figure. The whole was made fast to the axis of a convenient rotary apparatus, so that the whole could be put in motion about the central magnetic axis (common to both magnets). The ends $e$ and $f$ of the wire loop were brought out and attached separately to two insulated metal rings fastened on the axis of rotation, and suitable springs pressed upon these rings, these springs thus serving to carry off any current to a conveniently placed galvanometer (Phil. Trans. 1852, p. 30, \&c.). The whole arrangement is accordingly very simple. The magnets with rigidly attached rectangular loop were revolved together (as one whole) about the central axis, and no current or deflection was observed at the galvanometer. The absence of current was explained in the following manner. The outer projecting part of the rectangular loop, viz. a $d e$, was supposed by its rotation to cut through the outer lines of force of the magnet, which were considered to remain in absolute rest while the magnet revolved. The inner part of the rectangular loop, viz. $a b f$ (dotted in the diagram), was supposed, on the other hand, to cut through the inner or interior magnetic lines of force, which were also thought to remain in rigid rest, while the (compound) magnet independently revolved through them. In this way it was supposed that two opposite currents tended to be produced in the rectangular loop, and so exactly neutralized each other; and the absence of current in the loop was thus explained. But instead of having recourse to this complicated hypothesis, one can explain the absence of current by the simple fact that the whole system is at rest when it revolves as a whole, or there is no relative motion between its parts, which are rigidly connected; and without relative motion there can be no inductive effect* .

* Faraday considered that this experiment-where magnet and attached wire loop are revolved as one rigid whole-proved that the lines of force within the magnet were equal in power to those outside the magnet; because he supposed that the lines of force remained in absolute rest while the system revolved through them, whereby the current generated by the intersection of the internal lines of force was considered equal and opposite to that generated by the intersection of the external lines of force. Faraday says :- "The results, when the wire and magnet rotated together, 
Moreover, it seems very apparent on consideration that when the above double magnet rotates, its lines of force must rotate with it. For imagine one of the two magnets (which form the compound bar) to be removed. Then the centre of gravity of the other will now lie outside the axis of rotation. Therefore this magnet and therefore its lines of force will (by the rotation) be bodily translated about the axis. For Faraday himself admits that when a magnet (or its centre of gravity) is translated, its lines of force accompany it, and would intersect and inductively affect external conductors. The sams reasoning now applies to both magnets (if the other be brought back), so that when the whole revolves the lines of force of each bar (and therefore of the whole) are transluted about the axis, i.e. revolve with the magnet (or compound bar), and would influence external conducting bodies. This must, I think, be sufficiently clear; indeed, by holding a sheet of paper with loose iron filings horizontally above the end of the compound bar, one could, no doubt, in a certain sense, see the movement of the lines of force by observing the small disturbances produced in the iron filings, as the irregularities in shape or magnetic distribution of the poles pass under the filings when the compound bar revolves. This must be obvious enough, and it will probably be thought superfluous to carry the analysis of this point further. For we are concerned here only with one single oversight (depending on a double or ambiguous aspect in the expcriments); and as soon as Faraday, in the first instance, thought his view warranted or necessitated by the experimental results, he, of course (consistently), applied it throughout, and so a considerable number of experiments are affected. My object is only to reach the truth, and, whatever it may be, we shall certainly gain by its recognition.

As regards the experiments of Plücker, although the various forms of apparatus specially constructed by him for the determination of this and related questions is generally more complicated than the apparatus of Faraday, yet it is easy to see on going through the drawings that the same oversight, viz. the neglect of the influence of the galvanometer wires (or the external galvanometer-loop circuit) on the results, affects all his experiments alike; so that $I$ deem it unnecessary to consider these experiments further here. They are described very completely in the paper in Poggen-

show that these (currents) are perfectly equal to each other" (p. 33). My contention is that this experiment proves nothing, although there may be independent grounds for concluding that the lines of force outside the magnet are equal in power to those inside.

Phil. Mag. S. 5. Vol. 19. No. 117. Feb. 1885. 
dorff's Annalen before referred to. I will only mention one point. In one of Plücker's experiments he used an apparatus consisting of a copper disk, $d$ (fig. 5), in which six round bar-magnets were fixed vertically, as the diagram shows. The whole was rotated about the central axis. It was then thought (according to the view adopted by him and previously held by Faraday) that the lines of force did not share the rotation* of each bar-magnet about its axis, although they did share the rotation, $i$. e. translation (in a circle), of each barmagnet about the axis of the disk when the latter revolved. Hence it was believed that when the whole system revolved, the lines of force of each magnet remained axially in absolute rest, and therefore (virtually) revolved backwards, relatively to the direction of revolution of the disk, thereby intersecting the disk and themselves, and charging up the disk inductively, as it was thought. One sees to what a complicated result this hypothesis leads. The inductive charge of the disk was thought to be proved by tonching the axis with one galvanometer wire, and the periphery of the disk with the other, when a deflection was observed. It was overlooked that this deflection is attributable to the passing of the magnets through the galvanometer-loop or circuit as the disk revolves, whereby the lines of force of the magnets successively intersect the wires.

If one wished to illustrate this subject further experimentally, it would be necessary to be on one's guard against a special case, which otherwise might lead to deceptions. This may be best seen by taking a particular example. We will suppose that a magnet, $m$ (fig. 6 ), taken for simplicity the shape of a sphere, is surrounded by a metallic covering, or shell, $h$, with an air-space between. At the poles of the spherical magnet, projections $n s$ reach through holes in the shell, by which the magnet can be put in rotation independently-or (when necessary) connected to earth, or to an electrometer. Then, by the rotation of the magnet-sphere about its magnetic axis $n s$, the lines of force (partaking of the motion, on my view) will intersect the metallic shell $h$, and therefore strive to generate a current there, such that the parts of the shell opposite the equator and poles of the magnet will be statically charged with the opposite electricities, whose sign will depend on the direction of rotation. The charge on the metallic shell is accordingly just the same as if the magnet had been fixed and

* The lines of force were supposed to behave in this respect as magnetic needles would do, if balanced on pins projecting from the axis or each magnet; then when the whole revolves, the needles (pointing steadily north and south) would virtually revolve backwards, or remain axially at rest in space. 
the shell had been rotated in the opposite direction about the magnet (or, on my view, the charge depends on the relative* motion between the magnet and shell, and not on which revolves). When the shell revolves about the magnet, the existence of the charge upon it is admitted, and is dependent on well-known principles; but when the magnet revolves, the existence of the charge upon the shell would have been denied by Faraday-although the relative motion (between magnet and shell) is the same in both cases. For Faraday considered that "no mere rotation of a magnet on its axis produces any inductive effect on circuits exterior to it." But Faraday thought that the rotation of a magnet on its axis could produce an inductive effect on itself, or that the magnet could become statically charged by "revolving amongst its own forces." Although I contend that Faraday's experiments do not prove this static charge, I think I can show how (from another cause) such a static charge could come on the magnet in the present special case (fig. 6 ) without revolution "amongst its own forces." For it is apparent that if a static charge is produced on the shell by the axial revolution of the magnet (whose lines of force partake of its motion and intersect the shell), then this charge will act by static electric induction-like a Leyden jar-across the layer of air, and call forth an opposite static charge (or distribution of electricity) on the corresponding parts of the magnet facing the shell. Unless this fact (deducible beforehand from the considerations here set forth) be kept in view, this peculiar static charge may mislead, and make one think that the magnet could here have become charged in no other possible way but by "revolving amongst its own forces."

Since the first draft of this paper was written, I have observed that this question is touched upon in the very complete work of Prof. Wiedemann (Die Lehre vom Galcanismus), where there are also some references, viz. to Beer, Pogg. Ann. Bd. xciv. S. 177 (1855); Nobili, Pogg. Ann. Bd. xxvii. S. 421 (1833), \&c. But on referring to these papers, I find that they practically leave the question open (or have done nothing to decide it), and I find no mention of the possible double (or ambiguous) meaning of Faraday's fundamental experiment, upon which it may be said the whole question hinges. And

* It is curious here to remark that, on Faraday's view (that the "system of power about the magnet must not be considered as revolving with the magnet"), it would follow that the inductive effect on the shell produced by revolving it concentrically through the lines of force of the magnet would not be altered if the magnet itself were put in motion at the same rate as the shell (so that the two were in relative rest); or it would be necessary to conclude from this view of Faraday's that the inductive effect between two bodies is the same by relative rest as by relative motion. 


\section{Electromagnetio Experiments of Faraday and Plücker.}

it would seem desirable that any diverse views on a tolerably fundamental question of electromagnetism should cease, or a satisfactory general agreement be arrived at; which may be facilitated by an unbiassed discussion of the subject in the interests of truth.

Subsequent Addendum.-Besides the practical aspect of this question as relating to the revolving earth (as a magnet), and to the identity in fundamental qualities between the magnet and the helix (involving the validity of Ampère's theory), there is another practical application of the matter.

It seems, namely, extremely difficult (if not impossible) on Faraday's view of " a singular independence of the magnetism and the bar in which it resides," to explain how a bar-magnet is caused to rotate on its axis when one terminal of a galvanic element is connected with one pole of the magnet, and the other terminal with the equatorial part of the magnet (the magnet being suspended freely, and the terminals of the galvanic cell maintained in sliding contact with its equator and pole). Thus, suppose the galvanometer, $g$, of fig. 3 to be removed, and a battery or galvanic element substituted for it. Then the magnet is observed to revolve (as is well known). But how is the magnet to be made to revolve by the action of the current on its lines of force if the lines of force are independent of (the rotary motion) of the magnet? The lines of force may be made to revolve no doubt (by the current); but how are they to drag the magnet round with them when they are independent of it?-indeed so independent (as Faraday supposes) that the magnet can be actually made to rotate through its own lines (or field of force). If the rotation of the magnet cannot cause the field of force to rotate, how can (conversely) the rotation of the field of force cause the magnet to rotate ; in other words, how is the rotation of the magnet by the action of a current to be accounted for on Faraday's hypothesis? It appears that, on the opposite hypothesis, the explanation is simple; viz. the rotation of the magnet is then easily referable to the reaction of the current in the external portion of the circuit upon the external lines of force of the magnet (whereby these external lines of force are caused to revolve), and, being dependent on the magnet, the magnet is caused to revolve with them.

Heatherfield, Bournemouth, Dec. 1883, and

Paris, February 1885. 